\title{
Imprinting Magnetic Information in Manganites with X Rays
}

\author{
M. Garganourakis, V. Scagnoli, S. W. Huang, and U. Staub* \\ Swiss Light Source, Paul Scherrer Institut, CH 5232 Villigen PSI, Switzerland \\ H. Wadati \\ Department of Applied Physics and Quantum-Phase Electronics Center (QPEC), University of Tokyo, Hongo, Tokyo 113-8656, Japan \\ M. Nakamura \\ Cross-Correlated Materials Research Group (CMRG), Advanced Science Institute, RIKEN, Wako 351-0198, Japan \\ V. A. Guzenko \\ Laboratory for Micro- and Nanotechnology, Paul Scherrer Institut, 5232 Villigen PSI, Switzerland
}

M. Kawasaki and Y. Tokura

Department of Applied Physics and Quantum-Phase Electronics Center (QPEC), University of Tokyo, Hongo, Tokyo 113-8656, Japan and Cross-Correlated Materials Research Group (CMRG), Advanced Science Institute, RIKEN, Wako 351-0198, Japan

(Received 3 May 2012; published 9 October 2012)

\begin{abstract}
The effect of $x$ rays on an orbital and charge ordered epitaxial film of a $\operatorname{Pr}_{0.5} \mathrm{Ca}_{0.5} \mathrm{MnO}_{3}$ is presented. As the film is exposed to $\mathrm{x}$ rays, the antiferromagnetic response increases and concomitantly the conductivity of the film improve. These results are discussed in terms of a persistent $x$-ray induced doping, leading to a modification of the magnetic structure. This effect allows writing electronic and magnetic information in the film and represents a novel way of manipulating magnetism.
\end{abstract}

DOI: 10.1103/PhysRevLett.109.157203

PACS numbers: 75.25. $-\mathrm{j}$, 75.47.Lx

Changing magnetic properties with an external stimuli is an important task in many of today's memory applications, and new ways to manipulate magnetic properties are of current interest. It is straightforward to use magnetic fields to change spin direction on the microscopic level; however, alternative methods to influence spins will offer new opportunities. One class of materials in which magnetic moments can be manipulated are multiferroics, where there is a significant coupling between static electric fields and the magnetization. This allows changing multiferroic domains, reversing the direction of the cycloidal spin order [1] or influencing the spin order by tilting some moments [2]. On the other hand, in the study of ultrafast magnetization dynamics, it has been shown that ultra short intense laser pulses can lead to a magnetization reversal [3] just by heating above $T_{C}$ in the presence of two sublattice magnetizations [4].

In this respect, systems where there is significant coupling between magnetic and/or electronic properties of the material are promising candidates for the use of other stimuli to manipulate the magnetic properties. Manganites are such a class of materials, where the charge, orbital, and spin degrees of freedom are strongly entangled creating a variety of phases and instabilities at its phase boundaries [5]. Temperature, doping, electric and magnetic fields, and electromagnetic radiation can all change their electronic properties. In particular, it has been shown that electromagnetic radiation can be used to manipulate the electronic and structural properties of perovskite-type manganites. On the ultra fast time scale, terahertz pulses can directly excite vibrational excitations leading to a metal-insulator transition [6] where structural phase transitions can be induced by optical pulses [7]. In certain compositions, these transitions are long-lived [8]. However, in most cases, changes occur as a direct consequence of the pulse and responses are not permanent. A permanent phase change can be induced by $\mathrm{x}$ rays, where it has been shown that hard $\mathrm{x}$-ray exposure can destroy the crystallographic superstructure of the charge and orbital ordered phase at low temperatures for $\operatorname{Pr}_{1-x} \mathrm{Ca}_{x} \mathrm{MnO}_{3}$ for $x$ close to the ferromagnetic (FM) insulating phase boundary [9]. Similar effects were also observed via electron radiation [10]. More generally, it is well-known that x-ray diffraction can easily break bonds and destroy crystal structure, a significant drawback, e.g., in protein crystallography [11]. That $\mathrm{x}$ rays can be used to change materials properties has also been demonstrated recently in cuprates [12], where they influence oxygen ordering and also in multiferroics, where it can be used to influence the cycloidal domain population of a multiferroic [13]. Here, we demonstrate a different and novel effect of $\mathrm{x}$-ray interaction with a magnetic solid. We demonstrate that exposing an epixtial film of a half doped manganite by $\mathrm{x}$ rays does change both its electronic state and its magnetic structure. This persistent effect allows us to imprint and erase magnetic and electronic information in a continuous way, solely in the exposed region.

In this Letter, we concentrate on $\operatorname{Pr}_{1-x} \mathrm{Ca}_{x} \mathrm{MnO}_{3}, 0.3 \leq$ $x \leq 0.5$, which is a bandwidth controlled insulator with a CE-type charge and orbital order (OO), forming zigzag 


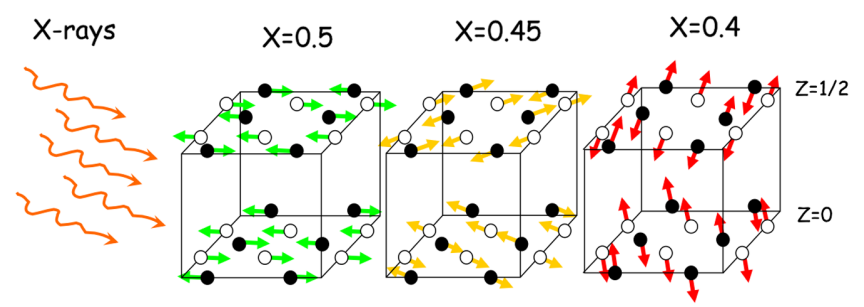

FIG. 1 (color online). Pictorial view of the $\mathrm{x}$-ray induced electron doping with canting of the manganese magnetic moments of $\operatorname{Pr}_{1-x} \mathrm{Ca}_{x} \mathrm{MnO}_{3}, 0.4 \leq x \leq 0.5$, and corresponding changes in the magnetic structure.

chains in the planes [14]. For $x=0.5$, the charge and $\mathrm{OO}$ sets in around $230 \mathrm{~K}$ and its usually associated with a change in conductivity. Below $T_{N}$ of $\sim 170 \mathrm{~K}$, the magnetic moments are antiferromagnetically ordered and lie fully in the $a b$ plane [15]. By lowering the doping level $x$, the moments rotate out of the plane and, around $x=0.3$, the system transforms into a FM insulator [15] as sketched here in Fig. 1. The magnetic transition temperature is reduced and the charge and $\mathrm{OO}$ is suppressed in the FM phase. To manipulate the magnetic state of the $\operatorname{Pr}_{0.5} \mathrm{Ca}_{0.5} \mathrm{MnO}_{3}$ thin film and measure its response, we use resonant $\mathrm{x}$-ray diffraction (RXD) at the Mn edge $2 p_{3 / 2}-3 d L_{3}$ transition. RXD is a very sensitive tool to study the magnetic and OO and has been already used extensively to study this thin film [16] and other doped $\mathrm{PrMnO}_{3}$ perovskites [17-19].

An epitaxial thin film of $\mathrm{Pr}_{0.5} \mathrm{Ca}_{0.5} \mathrm{MnO}_{3}$ was deposited onto an $\left(\mathrm{LaAlO}_{3}\right)_{0.3}-\left(\mathrm{SrAl}_{0.5} \mathrm{Ta}_{0.5} \mathrm{O}_{3}\right)_{0.7}$ substrate with $\left[\begin{array}{lll}0 & 1 & 1\end{array}\right]$ direction normal to the surface by pulsed laser deposition. The x-ray experiments were performed at the Swiss Light Source, Paul Scherrer Institut, using the RESOXS-endstation [20] at the SIM beam line [21]. Gold contacts spaced $700 \mu \mathrm{m}$ apart were deposited on the $\mathrm{Pr}_{0.5} \mathrm{Ca}_{0.5} \mathrm{MnO}_{3}$ film's surface with a $100 \mu \mathrm{m}$ gap between the electrodes to record the electrical conductivity. An x-ray beam of size $100 \times 50 \mu \mathrm{m}\left(\sim 6 \times 10^{12}\right.$ photons/s $)$ was centered into the gap, to simultaneously expose and collect the diffracted Bragg intensities. The measurement protocol is shown as an inset in Fig. 2. After an initial alignment and a heating and cooling cycle without $\mathrm{x}$-ray beam, the $\mathrm{x}$-ray intensity followed by the in situ electrical conductivity between the electrodes was collected (without $x$ rays impinging on the sample).

In Fig. 2, we present the dependence of the orbital and magnetic (1/4 1/4 0) Bragg peak intensity and the conductivity on $\mathrm{x}$-ray exposure. The polarization of the incident beam was set in the scattering plane, so called $\pi$-geometry. An incident $\mathrm{x}$-ray energy of $642.5 \mathrm{eV}$, and having the [-112] direction perpendicular to the scattering plane, was used.

Two remarkable phenomena are observed as a function of x-ray exposure: first, an increase in the conductivity and second, an enhancement of the magnetic or orbital Bragg peak intensity. The increase in conductivity might

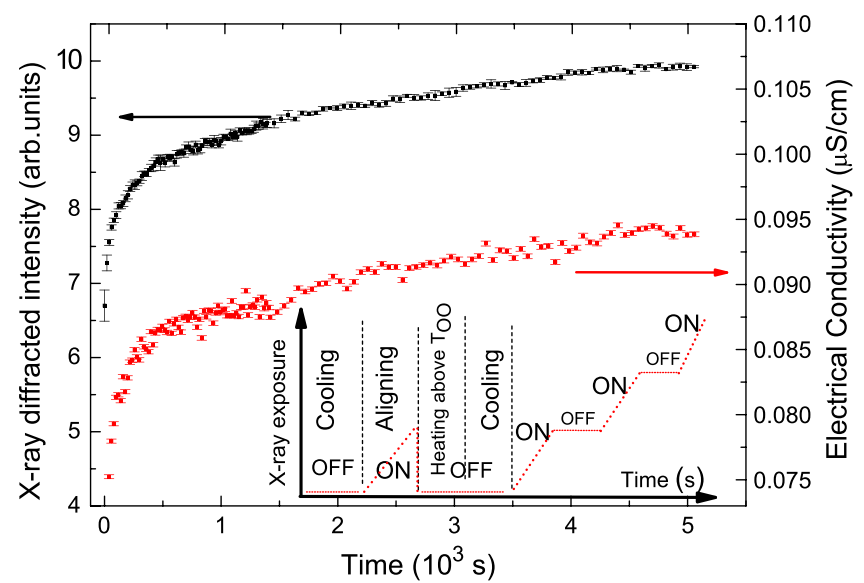

FIG. 2 (color online). X-ray exposure dependence of the $(1 / 41 / 40)$ reflection (left-hand side axis) and in situ collected electrical conductivity (right-hand side axis) of epitaxial $\operatorname{Pr}_{0.5} \mathrm{Ca}_{0.5} \mathrm{MnO}_{3}$ thin film at $T=50 \mathrm{~K}$. The energy of the X-ray beam was tuned to the resonance of $\mathrm{Mn} L_{3}$ edge $(642.5 \mathrm{eV})$ with incoming $\pi$ polarization. Inset: sketch of the experimental procedure for recording the x-ray induced changes of both the diffracted intensity and the photoconductivity.

be expected since past studies showed this effect on $\operatorname{Pr}_{1-x} \mathrm{Ca}_{x} \mathrm{MnO}_{3}$ for $x=0.3$, close to the phase boundary to the FM and insulating phase [9]. Surprisingly, the magnetic and orbital (1/4 1/4 0) peak intensity enhances as a function of $\mathrm{x}$-ray exposure time, in contrast to the suppression of the structural superlattice reflection observed in $\mathrm{Pr}_{0.7} \mathrm{Ca}_{0.3} \mathrm{MnO}_{3}$ [9]. In addition, the width of the Bragg peak decreases with increasing x-ray exposure, which directly relates to an increase in the correlation length of the antiferromagnetic or OO (Fig. 3 inset). The integrated intensity still increases as can be seen from the rocking curves in Fig. 3. In addition to the enhancement of the magnetic signal, the $\mathrm{x}$-ray illumination also improves the

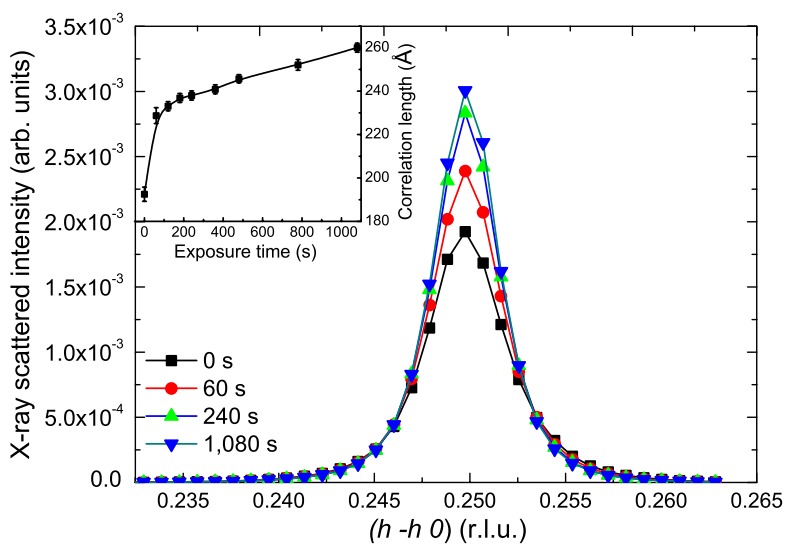

FIG. 3 (color online). Reciprocal space $(h-h 0)$ scan of the (1/4 1/4 0) reflection at x-ray energy $642.5 \mathrm{eV}$ using incident $\pi$ polarization, taken at $T=50 \mathrm{~K}$, at different $\mathrm{x}$-ray exposure time. Inset: evolution of the correlation length with respect to $\mathrm{x}$-ray exposure time obtained through a fit with a Lorentzian. 
antiferromagnetic and/or $\mathrm{OO}$ of the system despite its expected destructive effect on the crystalline structure.

What is the microscopic origin of this unexpected result? The (1/4 1/4 0) reflection has a magnetic and an orbital contribution $[17,18]$. The orbital scattering of these reflections is well understood and a signal occurs only in the rotated $\left(\pi \sigma^{\prime}\right)$ and $\left(\pi^{\prime} \sigma\right)$ polarization channels, each with equal an intensity [22]. Therefore, the magnetic scattering is on average larger in the $\pi$ incident channel since at resonance the $\sigma \sigma^{\prime}$ channel is forbidden. As the signal probed with $\pi$ polarization is increasing much stronger with X-ray exposure than the one probed by $\sigma$, we can associate the changes to be mainly of magnetic origin (see Fig. 1 of Supplemental Material [23]). Note that an increase in the orbital signal (improved order), results in a more effective carrier localization. Correspondingly, this would reduce the conductivity which is inconsistent with our observation. This excludes the possibility that the $\mathrm{x}$ rays solely improve the $\mathrm{OO}$ as this will make the material more insulating.

Therefore, we can consider solely the resonant magnetic scattering, which is dominated by electric dipole transitions (E1). The magnetic scattering amplitude can be written as shown in Ref. [24],

$$
F_{\boldsymbol{\epsilon} \boldsymbol{\epsilon}^{\prime}} \propto-i\left(\boldsymbol{\epsilon}^{\prime} \times \boldsymbol{\epsilon}\right) \cdot \boldsymbol{F}_{m},
$$

where $\boldsymbol{\epsilon}^{\prime}$ and $\boldsymbol{\epsilon}$ are unit vectors of the incident and scattered polarization of the $\mathrm{x}$ rays, respectively, and $\boldsymbol{F}_{m}$ is the magnetic structure factor.

To obtain quantitative information, we should calculate the magnetic structure factor

$$
\boldsymbol{F}_{m}=\sum_{j} \boldsymbol{m}_{j} e^{i \boldsymbol{r}_{j} \boldsymbol{q}},
$$

where $\mathbf{m}_{j}$ is a tensor representing the magnetic moment at site $j$ identified by $\mathbf{r}_{j}$ and $\mathbf{q}$ is the wave vector. The structural phase factors and direction of the moment results in $F_{m}=0$ for the (1/4 1/4 0) reflection for the magnetic structure of $\operatorname{Pr}_{0.5} \mathrm{Ca}_{0.5} \mathrm{MnO}_{3}$. For the canted structure of the lower doping levels [such as in Figs. 1(b) and 1(c)], the structure factor calculation results in $\boldsymbol{F}_{m}=\left(0,0, F_{m}\right)$ and only the $\mathrm{Mn}^{3+}$ ions contribute to the magnetic signal. This indicates that the enhancement of the magnetic peak intensity arises from the ferromagnetically coupled $c$ axis components of the $\mathrm{Mn}^{3+}$ spins in the CE-type antiferromagnet. The calculated value of the ratio between $\pi$ and $\sigma$ incident polarization Bragg intensities is consistent to the observed ratio for $T \leq T_{N}$ (See Fig. 2 in Supplemental Material [23]) for this model consideration.

The basic mechanisms for the observed increase of the magnetic moment components along the $c$ axis has to be discussed in relation to the observed increase in the conductivity. The increase in conductivity is expected to be directly related to the creation of defects in the charge and OO. The effect of indirect electric fields on the sample due to charging can be excluded as the application of
$10 \mathrm{KV} / \mathrm{cm}$ does not lead to an increase in scattering intensity. Such a scenario is consistent with the previously observed effect on the destruction of the corresponding crystallographic superstructure of the $x=0.3$ compound [9]. The creation of these defects excite deep donor levels which act as impurities in the material. This mechanism is quite similar to the behavior of the $D X$ centres in III-V semiconductors [25]. The x-ray-induced deep donor level impurities will be ionized and the corresponding electrons excited to the conduction band, resulting in the $\mathrm{x}$ rays photodoping the material. This doping mimics a decrease in $\mathrm{Ca}$ concentration. The strong coupling of the electrons to the manganite lattice leads to a metastable state, which includes a local deformation of the lattice due to the "orbital or charge" defect, consistent with the fact that when the beam is turned off no recovery occurs. This doping effect can also naturally explain the enhancement of the magnetic intensity. Decreasing the Ca concentration, as well as photodoping, leads to an increase in the spin canting in $\mathrm{Pr}_{1-x} \mathrm{Ca}_{x} \mathrm{MnO}_{3}$, as visualized in Fig. 1. For these materials, it is well-known that increasing electron doping leads to a more metallic state since it favors the double exchange interaction allowing electrons with neighboring parallel spins to hop. In contrast, electrons with antiferromagnetic spin alignments are frozen by the competing superexchange interaction. The increase of the $(1 / 41 / 4$ 0 ) magnetic reflection reflects the alignment of the FM spin component at least along the $c$ axis. The increase of the magnetic correlation length for increasing $x$-ray exposure is more difficult to understand. It might indicate that increasing the electron doping level would make the material being in a single magnetic state with larger sizes, whereas for $x=0.5$, both states may coexist, leading to smaller "nano" regions.

To test the spatial dimension of the effect, we present in Fig. 4 the contour plots of the collected x-ray intensity of the $(1 / 41 / 40)$ reflection by rastering the sample through the beam. For this measurement, we used lower x-ray photon flux $\left(3 \times 10^{12}\right.$ photons $\left./ \mathrm{s}\right)$ and less exposure time

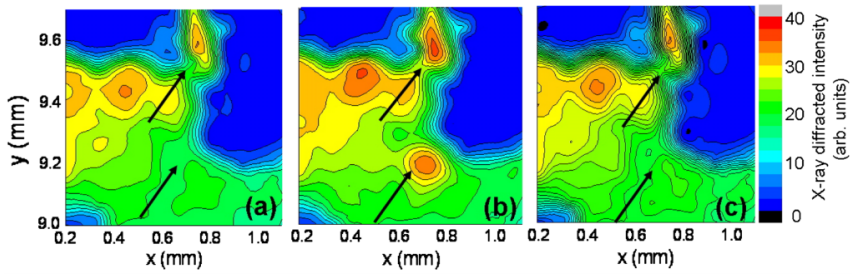

FIG. 4 (color online). Bragg peak intensity map of the $(1 / 4$ $1 / 40$ ) reflection of the $\operatorname{Pr}_{0.5} \mathrm{Ca}_{0.5} \mathrm{MnO}_{3}$ thin film at $T=50 \mathrm{~K}$. Before (a) and after (b) exposure to the $\mathrm{x}$ rays in the two positions (indicated by the arrows) for approximately 60 minutes. All experiments are performed at $50 \mathrm{~K}$ with $643.25 \mathrm{eV}$ with $\pi$ incident polarization. X-ray exposure effects were erased by warming up the sample above $T_{\mathrm{OO}}$ as shown in the pattern collected after cooling (c). 

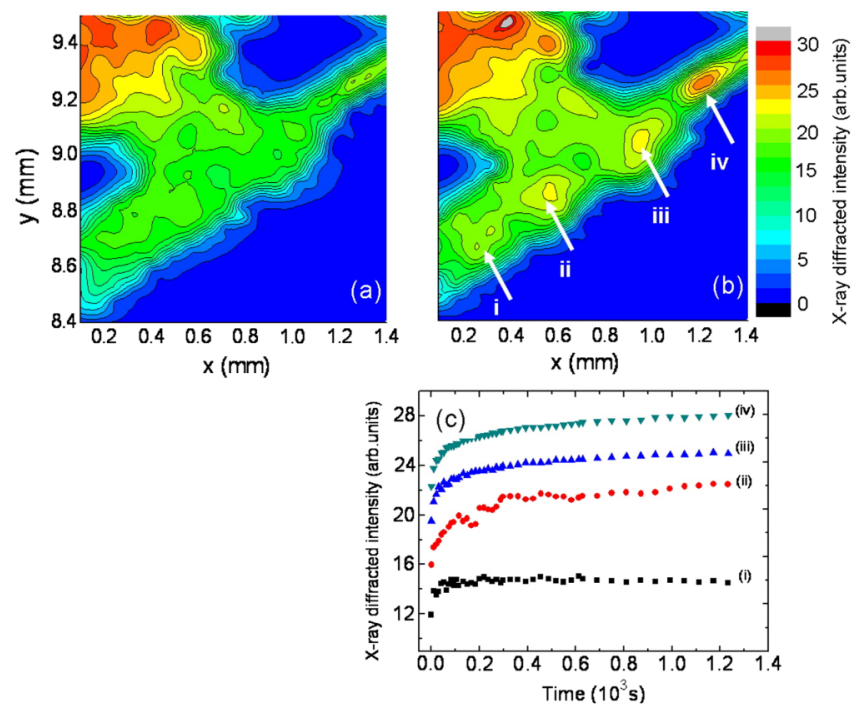

FIG. 5 (color online). Bragg peak intensity map of the $(1 / 4$ $1 / 40$ ) reflection of the $\operatorname{Pr}_{0.5} \mathrm{Ca}_{0.5} \mathrm{MnO}_{3}$ film at $T=50 \mathrm{~K}$ before (a) and after (b) exposing with different $\mathrm{x}$-ray conditions (see text) at four positions indicated by arrows, where in (c) the time dependence of the X-ray Bragg intensity at these positions is illustrated. The different curves are shifted to each other along the vertical axis for clarity. Writing the information in position (i), $3 \times 10^{12}$ photons/s were used with as beam size of $100 \times$ $50 \mu \mathrm{m}$ [21]. Position (ii) was exposed to $6 \times 10^{12}$ photons $/ \mathrm{s}$ where for positions (iii) and (iv), we exposed with $1.2 \times 10^{13}$ and $2.14 \times 10^{13}$ photons/s, respectively. The vertical spot size varied from 50 [position (i) and (ii)], to 100 [position (iii)], and $200 \mu \mathrm{m}$ for position (iv).

per point to reduce the effect of $x$ rays on the sample. The low intensity regions seen in Fig. 4 are locations where the sample is expected to be shadowed by the gold contacts and the indium wires. In order to write magnetic information, we have exposed two different portions of the sample to the x-ray beam (indicated by the arrows in Fig. 4). A clear increase of the scattered intensity is observed solely at the position of the x-ray exposure. After successfully writing magnetic information and its in situ observation, we tested the possibility to erase the written information. For this purpose, we heated the sample above the OO transition temperature $T_{\mathrm{OO}} \simeq 210 \mathrm{~K}$ and rastered the sample again after cooling back to $T=50 \mathrm{~K}$, Fig. 4(c). An intensity distribution identical to that before the $\mathrm{x}$-ray exposure was observed, Fig. 4(a), indicating a full recovery of the sample to the virgin state. This is also consistent with the view that the $\mathrm{x}$-ray induced defects are local charge and orbital defects in the electronic ordering pattern.

Finally, Fig. 5(a) displays a mesh scan of the virgin state of the film, whereas Fig. 5(b) shows the same region now exposed to four different spatially segregated x-ray flux conditions. The difference between positions (i) and (ii) represents a change of x-ray flux density by a factor of two, whereas the differences between (ii), (iii), and (iv), represent a change of exit slit of the beam line, leading to an increase in vertical spot size. The time dependence of the low flux density exposure for position (i) is very different from the others showing clear saturation behavior after 100 s, Fig. 5(c). This indicates that the process of interaction between the observed canting of the magnetic moments by $\mathrm{x}$ rays is a strongly nonlinear process.

To summarize, in this Letter, we describe a novel way to imprint magnetic and electronic information in strongly correlated manganites. The $\mathrm{x}$-ray illumination photodopes an epitaxial thin film of $\operatorname{Pr}_{0.5} \mathrm{Ca}_{0.5} \mathrm{MnO}_{3}$ by creating defects in the magnetic and orbital ordered state, improving the long range order associated with the antiferromagnetic state. As a result, the manganese magnetic moments realign from a collinear antiferromagnetic structure to a canted structure with FM magnetic components along the $c$ axis. As x rays can be focused down to the sub micrometer level, it could represent a new way for writing simultaneous magnetic and electronic information into a material. Moreover, the strength of changes can be varied by either tuning the $\mathrm{x}$-ray flux density or by changing the exposure time. This novel effect is not only interesting in the understanding of the physics of strongly correlated systems, but it may also offer a novel route to manipulate magnetism.

The authors would like to acknowledge the beam line staff for the vital support as well as Dr. P. M. Derlet for improving the text style. This work has been supported by the Swiss National Science Foundation and its National Centre of Competence of Research-Materials with Novel Electrical Properties and the Japan Society for the Promotion of Science (JSPS) through the "Funding Program for World-Leading Innovative R\&D on Science and Technology (FIRST Program)", initiated by the Council for Science and Technology Policy (CSTP).

*urs.staub@psi.ch

[1] Y. Yamasaki, H. Sagayama, T. Goto, M. Matsuura, K. Hirota, T. Arima, and Y. Tokura, Phys. Rev. Lett. 98, 147204 (2007).

[2] Y. Bodenthin, U. Staub, M. García-Fernández, M. Janoschek, J. Schlappa, E. I. Golovenchits, V. A. Sanina, and S. G. Lushnikov, Phys. Rev. Lett. 100, 027201 (2008).

[3] A. V. Kimel, A. Kirilyuk, P. A. Usachev, R. V. Pisarev, A. M. Balbashov, and T. Rasing, Nature (London) 435, 655 (2005).

[4] T. A. Ostler et al., Nature Commun. 3, 666 (2012).

[5] Y. Tokura, Colossal Magnetoresistive Oxides (Gordon and Breach Science Publishers, Amsterdam, 2000), Chap. 1.

[6] M. Rini, R. Tobey, N. Dean, J. Itatani, Y. Tomioka, Y. Tokura, R.W. Schoenlein, and A. Cavalleri, Nature (London) 449, 72 (2007).

[7] P. Beaud, S. L. Johnson, E. Vorobeva, U. Staub, R. A. De Souza, C. J. Milne, Q.X. Jia, and G. Ingold, Phys. Rev. Lett. 103, 155702 (2009).

[8] N. Takubo, Y. Ogimoto, M. Nakamura, H. Tamaru, M. Izumi, and K. Miyano, Phys. Rev. Lett. 95, 017404 (2005). 
[9] V. Kiryukhin, D. Casa, J. P. Hill, B. Keimer, A. Vigliante, Y. Tomioka, and Y. Tokura, Nature (London) 386, 813 (1997).

[10] Y. Horibe, C. H. Chen, S.-W. Cheong, and S. Mori, Europhys. Lett. 70, 383 (2005).

[11] E. F. Garman and R. L. Owen, Acta Crystallogr. Sect. D 62, 32 (2006).

[12] N. Poccia, M. Fratini, A. Ricci, G. Campi, L. Barba, A. Vittorini-Orgeas, G. Bianconi, G. Aeppli, and A. Bianconi, Nature Mater. 10, 733 (2011).

[13] E. Schierle, V. Soltwisch, D. Schmitz, R. Feyerherm, A. Maljuk, F. Yokaichiya, D. N. Argyriou, and E. Weschke, Phys. Rev. Lett. 105, 167207 (2010).

[14] J. B. Goodenough, Phys. Rev. 100, 564 (1955).

[15] Z. Jirak, S. Krupička, Z. Šimša, M. Dlouhă, and S. Vratislav, J. Magn. Magn. Mater. 53, 153 (1985).

[16] H. Wadati, J. Geck, E. Schierle, R. Sutarto, F. He, D. G. Hawthorn, M. Nakamura, M. Kawasaki, Y. Tokura, and G. A. Sawatzky, arXiv:1111.4725v1.

[17] K. J. Thomas, J.P. Hill, S. Grenier, Y-J. Kim, P. Abbamonte, L. Venema, A. Rusydi, Y. Tomioka, Y. Tokura, D.F. McMorrow, G. Sawatzky, and M. van Veenendaal, Phys. Rev. Lett. 92, 237204 (2004).

[18] U. Staub, M. García-Fernández, Y. Bodenthin, V. Scagnoli, R. A. De Souza, M. Garganourakis, E. Pomjakushina, and K. Conder, Phys. Rev. B 79, 224419 (2009).
[19] S. Y. Zhou, Y. Zhu, M. C. Langner, Y.-D. Chuang, P. Yu, W. L. Yang, A. G. Cruz Gonzalez, N. Tahir, M. Rini, Y.-H. Chu, R. Ramesh, D.-H. Lee, Y. Tomioka, Y. Tokura, Z. Hussain, and R.W. Schoenlein, Phys. Rev. Lett. 106, 186404 (2011).

[20] U. Staub, V. Scagnoli, Y. Bodenthin, M. GarcíaFernández, R. Wetter, A. M. Mulders, H. Grimmer, and M. Horisberger, J. Synchrotron Radiat. 15, 469 (2008).

[21] U. Fleschig, F. Nolting, A. Fraile Rodríguez, J. Krempaský, C. Quitmann, T. Schmidt, S. Spielmann, and D. Zimoch, AIP Conf. Proc. 1234, 319 (2010).

[22] U. Staub, V. Scagnoli, A. M. Mulders, K. Katsumata, Z. Honda, H. Grimmer, M. Horisberger, and J. M. Tonnerre, Phys. Rev. B 71, 214421 (2005).

[23] See Supplemental Material at http://link.aps.org/ supplemental/10.1103/PhysRevLett.109.157203 for Fig. S1 with explanations that discuss the polarization dependence of the X-ray exposure effect and Fig. S2 which gives the temperature dependence for both incident $\mathrm{x}$-ray polarizations with further experimental details.

[24] J. P. Hannon, G. T. Trammell, M. Blume, and D. Gibbs, Phys. Rev. Lett. 61, 1245 (1988).

[25] D. V. Lang, in Deep centres in Semiconductors, edited by S. T. Pantelides (Gordon and Breach, New York, 1986), p. 489. 